Article

\title{
Asymmetries in Processes of Electron-Positron Annihilation
}

\author{
Andrej Arbuzov ${ }^{1,2, *,+}+\mathbb{D}$, Serge Bondarenko ${ }^{1,+}(\mathbb{D})$ and Lidia Kalinovskaya ${ }^{3,+}(\mathbb{D}$ \\ 1 Bogoliubov Laboratory of Theoretical Physics, JINR, Joliot-Curie str. 6, 141980 Dubna, Russia; \\ bondarenko@jinr.ru \\ 2 Dubna State University, Universitetskaya str. 19, 141982 Dubna, Russia \\ 3 Dzhelepov Laboratory of Nuclear Problems, JINR, Joliot-Curie str. 6, 141980 Dubna, Russia; \\ lidia.kalinovskaya@cern.ch \\ * Correspondence: arbuzov@theor.jinr.ru \\ $\dagger$ These authors contributed equally to this work.
}

Received: 14 June 2020; Accepted: 6 July 2020; Published: 7 July 2020

check for updates

\begin{abstract}
Processes of electron-positron annihilation into a pair of fermions were considered. Forward-backward and left-right asymmetries were studied, taking into account polarization of initial and final particles. Complete 1-loop electroweak radiative corrections were included. A wide energy range including the $Z$ boson peak and higher energies relevant for future $e^{+} e^{-}$colliders was covered. Sensitivity of observable asymmetries to the electroweak mixing angle and fermion weak coupling was discussed.
\end{abstract}

Keywords: high energy physics; electron-positron annihilation; forward-backward asymmetry; left-right asymmetry

PACS: 12.15.-y; 12.15.Lk; 13.66.Jn

\section{Introduction}

Symmetries play a key role in the construction of physical theories. In fact, they allow us to describe a huge variety of observables by means of compact formulae. We believe that the success of theoretical models based on symmetry principles is due to the presence of the corresponding properties in Nature. The Standard Model (SM) is the most successful physical theory ever. Its predictions are in excellent agreement with practically all experimental results in particle physics. The renormalizability of the model allows us to preserve unitarity and provide finite verifiable results. Both phenomenological achievements and nice theoretical features of the SM are mainly due to the extended usage of symmetries in its construction. The model is based on several symmetries of different type, including the Lorentz (Poincaré) symmetry, the gauge $S U(3)_{C} \times S U(2)_{L} \times U(1)_{Y}$ symmetries, the CPT symmetry, the spontaneously broken global $S U(2)_{L} \times S U(2)_{R}$ symmetry in the Higgs sector, etc. Some symmetries of the model are exact (or seem to be exact within the present precision) while others are spontaneously or explicitly broken. In particular, the nature of the symmetry among the three generations of fermions is one of the most serious puzzles in the SM and verification of the lepton universality hypothesis is on the task list of modern experiments.

Despite the great successes of the SM, we can hardly believe that it is the true fundamental theory of Nature. Most likely, it is an effective model with a limited applicability domain. The search for the upper energy limit of the SM applicability is the actual task at all high-energy colliders experiments. Up to now, all direct attempts to find elementary particles and interactions beyond the Standard Model have failed. The accent of experimental studies has shifted towards accurate verification of the SM features. Deep investigation of the SM symmetries is an important tool in this line of research. 
Asymmetries form a special class of experimental observables. First of all, they explicitly access the breaking of a certain symmetry in Nature. Second, they are usually constructed as a ratio of observed quantities, in which the bulk of experimental and theoretical systematic uncertainties is canceled out. So the asymmetries provide independent additional information on particle interactions. They are especially sensitive to non-standard weak interactions including contributions of right currents and new intermediate $Z^{\prime}$ vector bosons, see e.g., [1].

The physical programs of future (super) high-energy electron-positron colliders such as CLIC [2], ILC [3-5], FCC-ee [6], and CEPC [7] necessarily include accurate tests of the SM. Studies of polarization effects and asymmetries will be important to probe of the fundamental properties of Higgs boson(s) and, in particular, in the process of annihilation into top quarks [8-10]. The future colliders plan to start operation in the so-called GigaZ mode at the $Z$ peak and improve upon the LEP both in statistical and systematical uncertainties in tests of the SM [11] by at least one order of magnitude. Among these collider projects, the FCC-ee one has the most advanced program of high-precision measurements of SM processes at the $Z$ peak. Such tests have been performed at LEP and SLC and they have confirmed the validity of the SM at the electroweak (EW) energy scale of about $100 \mathrm{GeV}[12,13]$. During the LEP era, extensive experimental and theoretical studies of asymmetries made an important contribution to the overall verification of the SM, see review [14] and references therein. The new precision level of future experiments motivates us to revisit the asymmetries and scrutinize the effects of radiative corrections (RCs) to them. In the analysis of LEP data, semi-analytic computer codes like ZFITTER [15] and TOPAZO [16] were extensively used. The forthcoming new generation of experiment requires more advanced programs, primarily Monte Carlo event generators.

The article is organized as follows. The next section contains preliminary remarks and the general notations. Section 3 is devoted to the left-right asymmetry. The forward-backward asymmetry is considered in Section 4. Discussion of the left-right forward-backward asymmetry is presented in Section 5. In Section 6, we provide results related to the final state fermion polarization. Section 7 contains a discussion and conclusions.

\section{Preliminaries and Notations}

In the recent paper [17] by the SANC group, high-precision theoretical predictions for the process $e^{+} e^{-} \rightarrow l^{+} l^{-}(l=\mu$ or $\tau)$ were presented. With the help of computer system SANC [18], we calculated the complete 1-loop electroweak radiative corrections to these processes, taking into account possible longitudinal polarization of the initial beams. The calculations were performed within the helicity amplitude formalism, taking into account the initial and final state fermion masses. So, the SANC system provides a solid framework to access asymmetries in $e^{+} e^{-}$annihilation processes and to study various relevant effects. In particular, the system allows us to separate effects due to quantum electrodynamics (QED) and weak radiative corrections.

The focus of this article is on the description and assessment of the asymmetry family: the left-right asymmetry $A_{\mathrm{LR}}$, the forward-backward asymmetry $A_{\mathrm{FB}}$, the left-right forward-backward asymmetry $A_{\mathrm{LRFB}}$, and the final state fermion polarization $P_{\tau}$ in collisions of high-energy polarized or unpolarized $e^{+} e^{-}$beams. The main aim was to verify the effect of radiative corrections on the extraction of the SM parameters from the asymmetries and to analyze the corresponding theoretical uncertainty.

We performed calculations for polarized initial and final state particles. Beam polarizations play an important role:

- They improve the sensitivity to CP-violating anomalous couplings or form factors, which are measurable even with unpolarized beams through the forward-backward asymmetry.

- With the polarization of both beams, the sensitivity to the new physics scale can be increased by a factor of up to 1.3 with respect to the case with only polarized electrons [1].

- A high-luminosity at the GigaZ stage of a collider running at the $Z$ boson resonance with positron polarization allows us to improve the accuracy of the determination of $\sin ^{2} \vartheta_{W}\left(\vartheta_{W}\right.$ is 
the electroweak mixing angle) by an order of magnitude, through studies of the left-right asymmetry [1].

Numerical illustrations for each asymmetry are given in two energy domains: the wide center-of-mass energy range $20 \leq \sqrt{s} \leq 500 \mathrm{GeV}$ and the narrow one around the $Z$ resonance $(70 \leq \sqrt{s} \leq 100 \mathrm{GeV})$, where a peculiar behavior of observables can be seen. All results were produced with the help of the $e^{+} e^{-}$branch [19] of the MCSANC Monte Carlo integrator [20].

Let us introduce the notation. First of all, we define quantities $A_{f}(f=e, \mu, \tau)$ which are often used for description of asymmetries at the $Z$ peak:

$$
A_{f} \equiv 2 \frac{g_{V_{f}} g_{A_{f}}}{g_{V_{f}}^{2}+g_{A_{f}}^{2}}=\frac{1-\left(g_{R_{f}} / g_{L_{f}}\right)^{2}}{1+\left(g_{R_{f}}^{2} / g_{L_{f}}^{2}\right)^{2}},
$$

where the vector and axial-vector coupling constants of the weak neutral current of the fermion $f$ with the electromagnetic charge $q_{f}$ (in the units of the positron charge $e$ ) are

$$
g_{V_{f}} \equiv I_{f}^{3}-2 q_{f} \sin ^{2} \vartheta_{W}, \quad g_{A_{f}} \equiv I_{f}^{3} .
$$

The corresponding left and right fermion couplings are

$$
g_{L_{f}} \equiv I_{f}^{3}-q_{f} \sin ^{2} \vartheta_{W}, \quad g_{R_{f}} \equiv-q_{f} \sin ^{2} \vartheta_{W} .
$$

The neutral current couplings $g_{L_{f}}$ and $g_{R_{f}}$ quantify the strength of the interaction between the $Z$ boson and the given chiral states of the fermion.

We claim that there are sizable corrections to all observable asymmetries due to radiative corrections which affect simple Born-level analytic formulae relating the asymmetries with electroweak parameters. It is especially interesting to consider the behavior of asymmetries in different EW schemes: $\alpha(0), \alpha\left(M_{Z}^{2}\right)$, and $G_{\mu}$, see their definitions below. We also will compare the results in the Born and 1-loop approximation. The latter means inclusion of 1-loop radiative corrections of one of the following types: pure QED photonic RCs (marked as "QED"), weak RCs (marked as "weak"), and the complete 1-loop electroweak RCs (marked as "EW"):

$$
\sigma_{\mathrm{EW}}=\sigma_{\mathrm{Born}}+\sigma_{\mathrm{QED}}+\sigma_{\text {weak }} .
$$

The weak part in our notation includes 1-loop self-energy corrections to photon and $Z$ boson propagators. In our notation, higher-order effects due to interference of pure QED and weak contributions are a part of $\sigma_{\text {weak }}$.

The cross section of a generic annihilation process of longitudinally polarized $e^{+}$and $e^{-}$with polarization degrees $P_{e^{+}}$and $P_{e^{-}}$can be expressed as follows:

$$
\begin{aligned}
\sigma\left(P_{e^{-}}, P_{e^{+}}\right) & =\left(1+P_{e^{-}}\right)\left(1+P_{e^{+}}\right) \sigma_{R R}+\left(1-P_{e^{-}}\right)\left(1+P_{e^{+}}\right) \sigma_{L R} \\
& +\left(1+P_{e^{-}}\right)\left(1-P_{e^{+}}\right) \sigma_{R L}+\left(1-P_{e^{-}}\right)\left(1-P_{e^{+}}\right) \sigma_{L L} .
\end{aligned}
$$

Here $\sigma_{a b}=\sum_{i j(k)}\left|\mathcal{H}_{a b i j(k)}\right|^{2}$ are the $2 \rightarrow 2(3)$ helicity amplitudes of the reaction, $(a b=R R, R L, L R, L L)$ with right-handed $R=$ " + " or left-handed $L="$ " " initial particles.

It is convenient to combine the electron $P_{e^{-}}$and positron $P_{e^{+}}$polarizations into the effective quantity

$$
P_{\text {eff }}=\frac{P_{e^{-}}-P_{e^{+}}}{1-P_{e^{-}} P_{e^{+}}} .
$$

In the case when only the electron beam is polarized, the effective polarization coincides with the electron one.

To investigate theoretical uncertainties, we use the following three EW schemes: 
1. the $\alpha(0)$ scheme in which the fine-structure constant $\alpha(0)$ is used as input. The contribution of RCs in this scheme is enhanced by the large logarithms of light fermion masses via $\alpha(0) \ln \left(s / m_{f}^{2}\right)$ terms.

2. The $\alpha\left(M_{Z}^{2}\right)$ scheme in which the effective electromagnetic constant $\alpha\left(M_{Z}^{2}\right)$ is used at Born level while virtual 1-loop and real photon bremsstrahlung contributions are proportional to $\alpha^{2}\left(M_{Z}^{2}\right) \alpha(0)$. In this scheme the virtual RCs receive contributions from the quantity $\Delta \alpha\left(M_{Z}^{2}\right)$ which describes the evolution of the electromagnetic coupling from the scale $Q^{2}=0$ to the $Q^{2}=M_{Z}^{2}$ one and cancels the large terms with logarithms of light fermion masses.

3. the $G_{\mu}$ scheme in which the Fermi coupling constant $G_{\mu}$, extracted from the muon life time, is used at the Born level while the virtual 1-loop and real photon bremsstrahlung contributions are proportional to $G_{\mu}^{2} \alpha(0)$. The virtual RCs receive contributions from the quantity $\Delta r$. Since the expression for $\Delta r$ contains the $\Delta \alpha\left(M_{Z}^{2}\right)$, the large terms with logarithms of the light masses are also canceled. The quantity $\Delta r$ rules the $G_{\mu}$ and $\alpha(0)$ relation in this scheme.

Results of fixed-order perturbative calculations in these schemes differ due to missing higher-order effects. In what follows, numerical calculations are performed in the $\alpha(0)$ EW scheme if another choice is not explicitly indicated.

\section{Left-Right Asymmetry $A_{\mathrm{LR}}$}

A scheme to measure the $A_{\mathrm{LR}}$ polarization asymmetry at the $Z$ peak was suggested in [21]. It was shown that this observable can be used as for extraction of electroweak couplings as well as for a polarimeter calibration.

If we neglect the initial electron masses, the polarized cross-section can be rewritten in the following form:

$$
\sigma\left(P_{e^{-}}, P_{e^{+}}\right)=\left(1-P_{e^{-}} P_{e^{+}}\right)\left[1-P_{\text {eff }} A_{\mathrm{LR}}\right] \sigma_{0}
$$

where $\sigma_{0}$ is the unpolarized cross-section.

The left-right asymmetry in the presence of partially polarized $\left(\left|P_{\text {eff }}\right|<1\right)$ initial beams is defined as

$$
A_{\mathrm{LR}}=\frac{1}{P_{\text {eff }}} \frac{\sigma\left(-P_{\text {eff }}\right)-\sigma\left(P_{\text {eff }}\right)}{\sigma\left(-P_{\text {eff }}\right)+\sigma\left(P_{\text {eff }}\right)}
$$

where $\sigma$ is the cross-section with polarization $P_{\text {eff }}$.

In the case of fully polarized initial particles $\left(\left|P_{e^{ \pm}}\right|=1\right)$ the definition (7) becomes:

$$
A_{\mathrm{LR}}=\frac{\sigma_{L_{e}}-\sigma_{R_{e}}}{\sigma_{L_{e}}+\sigma_{R_{e}}}
$$

where $L_{e}$ and $R_{e}$ refer to the left and right helicity states of the incoming electron.

Equations (6) and (7) show that $A_{\mathrm{LR}}$ does not depend on the degree of the initial beam polarization.

This type of asymmetry is sensitive to weak interaction effects in the initial vertex. In the Born approximation at energies close to the $Z$ resonance, it is directly related to the electron coupling:

$$
A_{\mathrm{LR}} \approx A_{e} .
$$

The left-right asymmetry $A_{\mathrm{LR}}$ as a function of the center-of-mass system (c.m.s.) energy in the ranges $20 \leq \sqrt{s} \leq 500 \mathrm{GeV}$ (Left) and $70 \leq \sqrt{s} \leq 110 \mathrm{GeV}$ (Right) is shown in Figure 1. We explore $A_{\mathrm{LR}}$ in different approximations and the corresponding shifts $\Delta A_{\mathrm{LR}}$ between the Born level and 1-loop corrected approximations taking into account either pure QED, or weak, or complete EW effects: $\Delta A_{\mathrm{LR}}=A_{\mathrm{LR}}$ (1-loop corrected)- $A_{\mathrm{LR}}$ (Born). The right figure shows the behavior of $A_{\mathrm{LR}}$ near the $\mathrm{Z}$ resonance, and the value $A_{e}$ at $\sqrt{s}=M z$ is indicated by a black dot (see (9)). 
One can notice that although the total 1-loop EW corrections to the process cross-section are equal to the sum of the pure QED and weak ones, the corresponding shifts $\Delta A_{\mathrm{LR}}$ are not additive. That is because the asymmetry is defined as a ratio and the corrections affect both the numerator and denominator.

In Figure 2 we show $A_{\mathrm{LR}}$ for the Born and weak 1-loop corrected levels of accuracy in different EW schemes and the corresponding shifts $\Delta A_{\mathrm{LR}}=A_{\mathrm{LR}}$ (weak, some EW scheme)- $A_{\mathrm{LR}}$ (Born). We see that the effects due to weak corrections in different EW schemes behave in a similar way. Nevertheless the scheme dependence is visible within the expected precision of future measurements. The deviations between the results in different schemes can be treated as a contribution into the theoretical uncertainty due to missing higher order corrections.
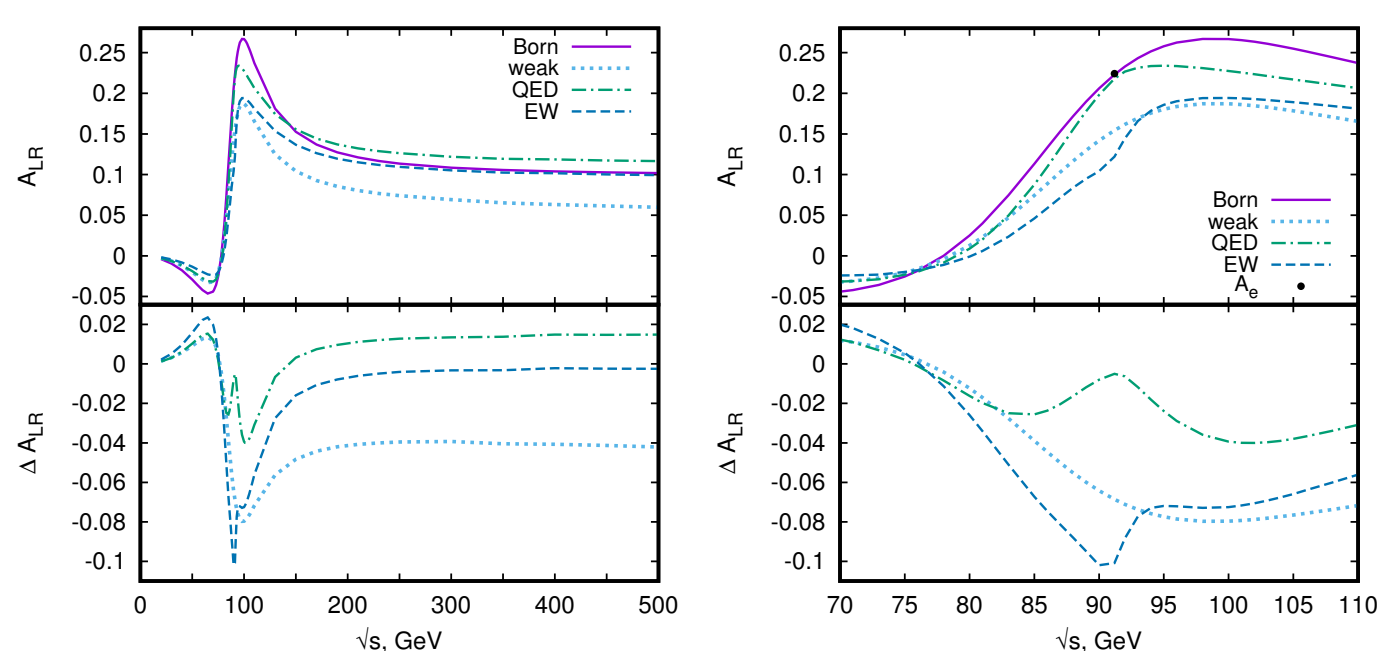

Figure 1. (Left) The $A_{\mathrm{LR}}$ asymmetry in the Born and 1-loop (weak, pure quantum electrodynamics (QED), and electroweak (EW)) approximations and $\Delta A_{\mathrm{LR}}$ vs. center-of-mass system (c.m.s.) energy in a wide range; (Right) the same for the $Z$ peak region.

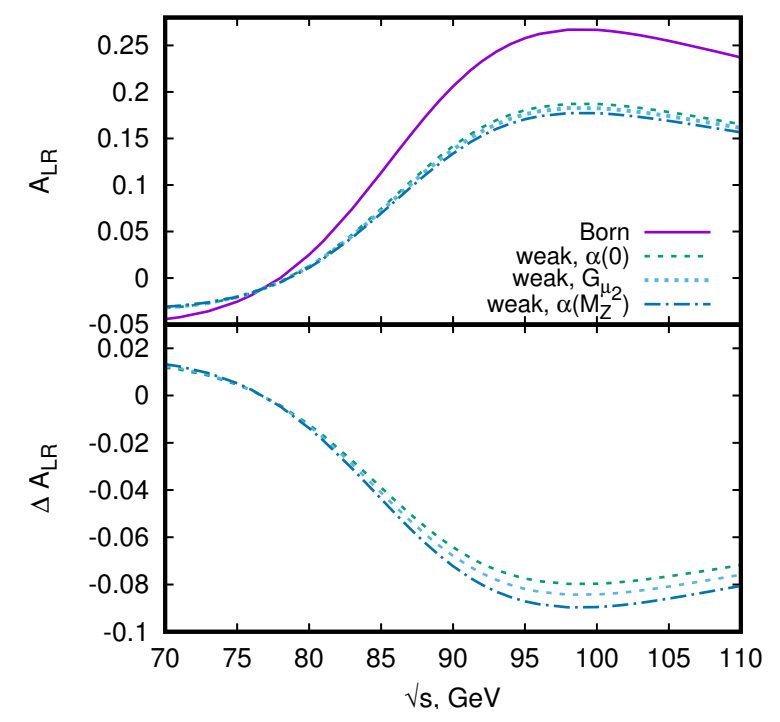

Figure 2. The $A_{\mathrm{LR}}$ asymmetry at the Born level and with 1-loop weak radiative corrections (RCs); the corresponding shifts $\Delta A_{\mathrm{LR}}$ within $\alpha(0), G_{\mu}$, and $\alpha\left(M_{\mathrm{Z}}^{2}\right)$ EW schemes vs. c.m.s. energy in the peak region.

The impact of 1-loop EW contributions to $\Delta A_{\mathrm{LR}}$ is of the order -0.1 in the resonance region, but at energies above $\sqrt{s}=200 \mathrm{GeV}$ there are considerable cancellations between weak and QED effects so that the combined EW corrections becomes small (but still numerically important for high-precision measurements). 


\section{Summary for $A_{\mathrm{LR}}$}

The left-right asymmetry $A_{\mathrm{LR}}$ is almost insensitive to the details of particle detection since the corresponding experimental uncertainties tend to cancel out in the ratio (7). It (almost) does not depend on the final state fermion couplings in the vicinity of the $Z$ boson peak and can be measured for any final state with a large gain in statistics. For this reasons it is appropriate for extraction of the $\sin ^{2} \vartheta_{W}^{\text {eff }}$ value.

We observe that the values $\Delta A_{\mathrm{LR}}$ due to weak and pure QED 1-loop corrections are very significant at high energies in general, but in the resonance region impact of QED is small, while the weak contribution to $\Delta A_{\mathrm{LR}}$ reaches 0.07 . Therefore, it is necessary to evaluate all possible radiative correction contributions to the weak parts of RCs carefully and thoroughly.

\section{Forward-Backward Asymmetry $A_{\mathrm{FB}}$}

The forward-backward asymmetry is defined as

$$
\begin{aligned}
& A_{\mathrm{FB}}=\frac{\sigma_{\mathrm{F}}-\sigma_{\mathrm{B}}}{\sigma_{\mathrm{F}}+\sigma_{\mathrm{B}}} \\
& \sigma_{\mathrm{F}}=\int_{0}^{1} \frac{d \sigma}{d \cos \vartheta_{f}} d \cos \vartheta_{f}, \quad \sigma_{\mathrm{B}}=\int_{-1}^{0} \frac{d \sigma}{d \cos \vartheta_{f}} d \cos \vartheta_{f},
\end{aligned}
$$

where $\vartheta_{f}$ is the angle between the momenta of the incoming electron and the outgoing negatively charged fermion. It can be measured in any $e^{+} e^{-} \rightarrow f \bar{f}$ channels but for precision test the most convenient channels are $f=e, \mu$. The channels with production of $\tau$ leptons, $b$ or $c$ quarks are very interesting as well.

At the Born level, this asymmetry is proportional to the product of initial and final state couplings and is caused by parity violation at both production and decay vertices:

$$
A_{\mathrm{FB}} \approx \frac{3}{4} A_{e} A_{f}
$$

In the case of partially polarized initial beams the condition (11) reduces to the following one

$$
A_{\mathrm{FB}} \approx \frac{3}{4} \frac{A_{e}-P_{\mathrm{eff}}}{1-A_{e} P_{\mathrm{eff}}} A_{f} .
$$

In Figure 3 we show the behavior of the $A_{\mathrm{FB}}$ asymmetry in the Born and 1-loop approximations (with weak, pure $\mathrm{QED}$, or complete $\mathrm{EW}$ contributions) and the corresponding $\Delta A_{\mathrm{FB}}$ for c.m.s. energy range $20 \leq \sqrt{s} \leq 500 \mathrm{GeV}$ in the left plot and for the $Z$ peak region of c.m.s. energy $70 \leq \sqrt{s} \leq 110 \mathrm{GeV}$ in the right one. As in the previous case of $A_{\mathrm{LR}}$, we indicate by a black dot the value $A_{\mathrm{FB}} \approx 3 / 4 A_{e} A_{\mu}$ at the resonance. We observe that the weak contribution to $A_{\mathrm{FB}}$ is small and practically does not depend on energy. The shift $\Delta A_{\mathrm{FB}}$ changes the sign at the resonance and tends to a constant value $(\sim-0.3)$ above $200 \mathrm{GeV}$. The huge magnitude of the shift $\Delta A_{\mathrm{FB}}$ out of the $Z$ resonance region is coming mainly from the pure QED corrections. In particular, above the peak the effect due to radiative return to the resonance is very important.

Figure 4 shows the dependence of $A_{\mathrm{FB}}$ for different levels of accuracy (Born and 1-loop weak) on the EW scheme choice: either $\alpha(0)$, or $\mathrm{G}_{\mu}$, or $\alpha\left(M_{Z}^{2}\right)$. The corresponding shifts $\Delta A_{\mathrm{FB}}$ between the Born and the 1-loop weak corrected approximations are shown in the lower plot. 

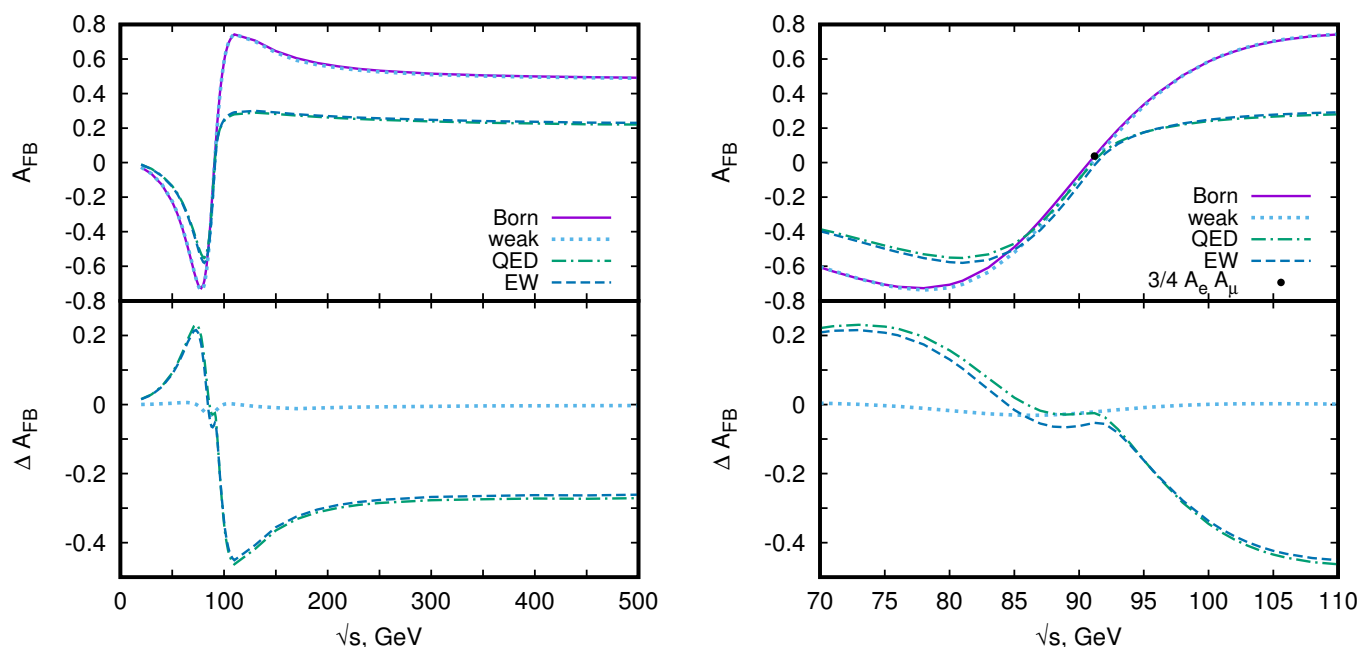

Figure 3. (Left) The $A_{\mathrm{FB}}$ asymmetry in the Born and 1-loop (weak, QED, EW) approximations and the corresponding shifts $\Delta A_{\mathrm{FB}}$ for a wide c.m.s. energy range; (Right) the same for the $Z$ peak region.

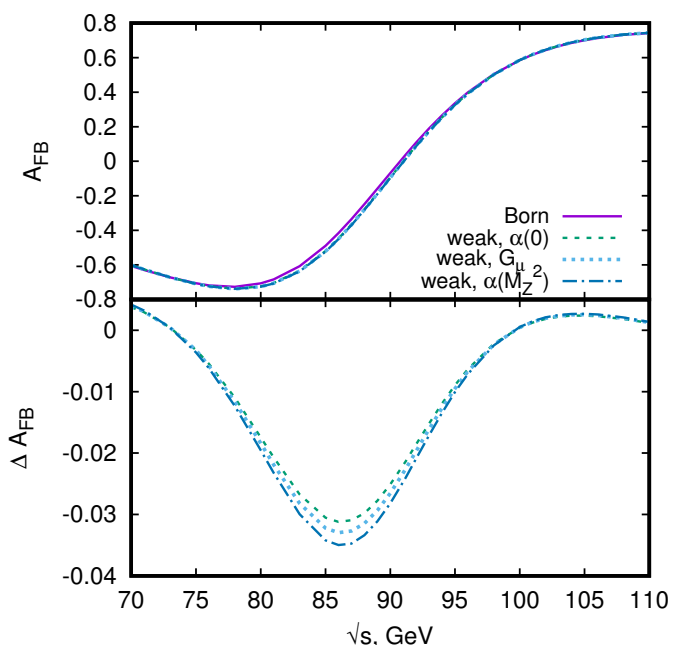

Figure 4. The $A_{\mathrm{FB}}$ asymmetry and $\Delta A_{\mathrm{FB}}$ in the Born and complete 1-loop EW approximations within the $\alpha(0), \mathrm{G}_{\mu}$, and $\alpha\left(M_{Z}^{2}\right)$ EW schemes vs. the c.m.s energy.

Below we investigate two sets of polarization degree $P_{i}=\left(P_{e^{-}}, P_{e^{+}}\right)$:

$$
P_{1}=(-0.8,0.3) \quad \text { and } \quad P_{2}=(0.8,-0.3) .
$$

In Figure 5 we compare the values of $A_{\mathrm{FB}}$ asymmetry and the corresponding shifts due to EW corrections for the unpolarized case and two choices of polarized beams defined in the above equation. One can see that a combination of polarization degrees of initial particles can either increase or decrease the magnitude of the $A_{\mathrm{FB}}$ asymmetry with respect to the unpolarized case.

There is an interesting idea [22] to use the $A_{\mathrm{FB}}$ asymmetry at the FCC-ee in order to directly access the value of QED running coupling at $M_{Z}$. This idea was supported in [23] where it was demonstrated that higher-order QED radiative corrections to $A_{\mathrm{FB}}$ are under control. Our results show that higher-order effects due to weak interactions are not negligible in this observable; further studies are required. 
At the Born level there are contributions suppressed by the small factor $m_{f}^{2} / s$ with the fermion mass squared. It is interesting to note that in 1-loop radiative corrections there are contributions of the relative order $\alpha \cdot m_{f} / \sqrt{s}$ with the fermion mass to the first power [24], which are numerically relevant at high energies especially for the $b$ quark channel.

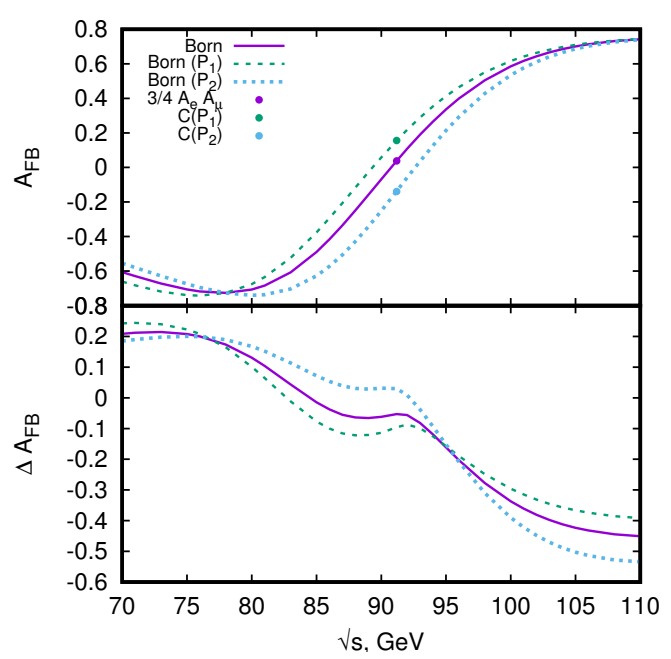

Figure 5. The $A_{\mathrm{FB}}$ asymmetry at the Born level (upper panel) and the corresponding $\Delta A_{\mathrm{FB}}$ in the 1-loop EW approximation (bottom panel) for unpolarized and polarized cases with degrees of beam polarizations $P_{1,2}(13)$ vs. c.m.s. energy in the $Z$ peak region. The constants $C\left(P_{1,2}\right)$ stand for the expression (12) with polarization degrees (13).

\section{Summary for $A_{\mathrm{FB}}$}

The weak 1-loop contribution $\Delta A_{\mathrm{FB}}$ is rather small for the whole energy range, see Figure 3. Nevertheless in this asymmetry the difference between the pure QED and the complete 1-loop approximations near the resonance is numerically important. The dependence on the EW scheme choice, see Figure 4, is small but still relevant for high-precision measurements. The dependence of this asymmetry on polarization is very significant.

\section{Left-Right Forward-Backward Asymmetry $A_{\text {LRFB }}$}

In order to measure the weak couplings of the final state fermions, it was suggested to analyze the so-called left-right forward-backward asymmetry [25]:

$$
A_{\mathrm{LRFB}}=\frac{\left(\sigma_{L_{e}}-\sigma_{R_{e}}\right)_{F}-\left(\sigma_{L_{e}}-\sigma_{R_{e}}\right)_{B}}{\left(\sigma_{L_{e}}+\sigma_{R_{e}}\right)_{F}+\left(\sigma_{L_{e}}+\sigma_{R_{e}}\right)_{B}},
$$

where $\sigma_{L}$ and $\sigma_{R}$ are the cross sections with left and right handed helicities of the initial electrons.

From the definition (14) it follows that $A_{\mathrm{LRFB}}$ partially inherits the properties of the $A_{\mathrm{LR}}$ and, in particular, does not depend on the degree of the initial beam polarizations.

In the case of unpolarized beams on the $Z$ resonance peak, the Born-level asymmetry is

$$
A_{\mathrm{LRFB}} \approx \frac{3}{4} A_{f}
$$

In Figure 6 we present the predictions for the $A_{\mathrm{LRFB}}$ asymmetry in several approximations, namely at the Born level and with 1-loop weak, pure QED, and complete EW contributions. 

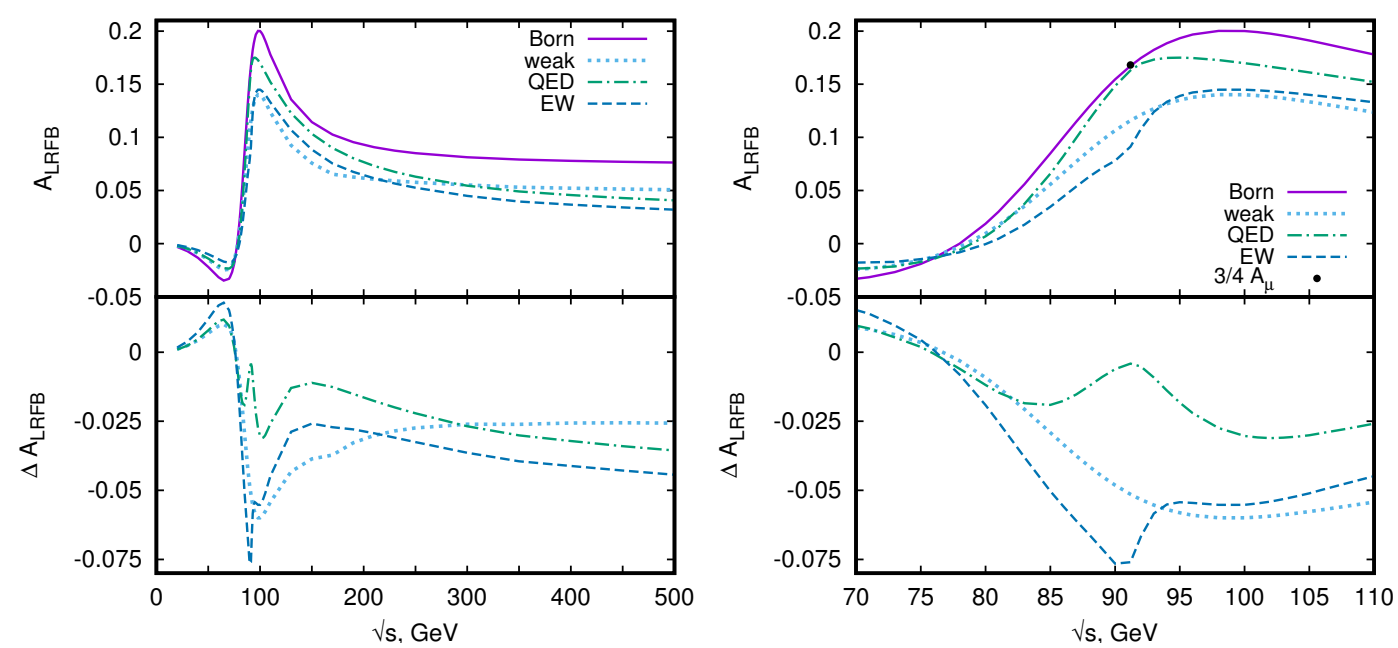

Figure 6. (Left) The $A_{\mathrm{LRFB}}$ asymmetry in the Born and 1-loop (weak, QED, EW) approximations and $\Delta A_{\mathrm{LRFB}}$ for c.m.s. energy range; (Right) the same for the $Z$ peak region.

Next, we repeat the study of the $A_{\text {LRFB }}$ asymmetry behavior in different EW schemes. We have illustrated the energy dependence of the $A_{\mathrm{LRFB}}$ asymmetry in $\alpha(0), \mathrm{G}_{\mu}$, and $\alpha\left(M_{\mathrm{Z}}^{2}\right)$ schemes and the corresponding $\triangle A_{\mathrm{LRFB}}$ in Figure 7 . The impact of weak corrections on $A_{\mathrm{LRFB}}$ is large. For example, the Born-level value of $A_{\mathrm{LRFB}}$ at the $Z$ peak is about 0.17 , while accounting for the weak RCs contribution reduces the asymmetry value down to $\sim 0.11$.

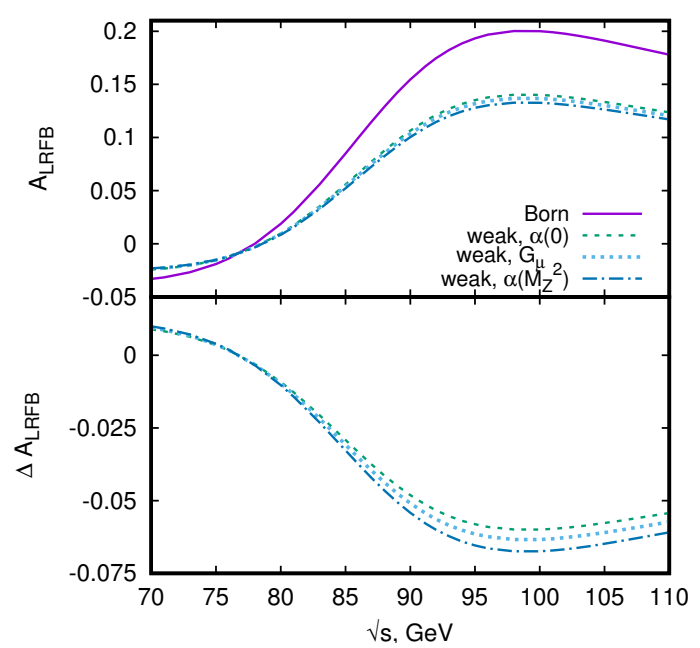

Figure 7. The $A_{\mathrm{LRFB}}$ asymmetry in the Born and 1-loop EW approximations and $\Delta A_{\mathrm{LRFB}}$ within $\alpha(0)$, $\mathrm{G}_{\mu}$, and $\alpha\left(M_{Z}^{2}\right)$ EW schemes vs. c.m.s. energy in the $Z$ peak region.

\section{Summary for $A_{\mathrm{LRFB}}$}

We would like to emphasize that the above Formula (15) appears to be a rather rough approximation since radiative corrections shift the observable value of $A_{\mathrm{LRFB}}$ quite a lot. Apparently the $A_{\mathrm{LRFB}}$ asymmetry is more affected by weak corrections than $A_{\mathrm{LR}}$. The shifts $\Delta A_{\mathrm{LRFB}}$ only slightly depend on an EW scheme choice. The $A_{\mathrm{LRFB}}$ asymmetry at the $Z$ boson peak depends on the final lepton coupling that could be used to measure the $\mu$ and $\tau$ weak couplings and their difference from the initial lepton (electron) one. 


\section{Final-State Fermion Polarization $\boldsymbol{P}_{f}$}

The polarization of a final-state fermion $P_{f=\mu, \tau}$ can be expressed as the ratio between the difference of the cross sections for right and left handed final state helicities and their sum

$$
P_{f}=\frac{\sigma_{R_{f}}-\sigma_{L_{f}}}{\sigma_{R_{f}}+\sigma_{L_{f}}} .
$$

In an experiment, it can be measured for the $\tau^{+} \tau^{-}$channel by reconstructing the $\tau$ polarization from the pion spectrum in the decay $\tau \rightarrow \pi v$. Details of the analysis of $P_{\tau}$ measurements at LEP are described in [13]. Computer programs TAOLA [26] and KORALZ [27,28] were applied for this analysis. Estimated improvement for $P_{\tau}$ and $\tau$ decay products over LEP time in ILC in the GigaZ program was done in [5].

In the case for unpolarized beams in the vicinity of the $Z$ peak, the expression for channel $e^{+} e^{-} \rightarrow \tau^{+} \tau^{-}$is simplified to

$$
P_{\tau}\left(\cos \vartheta_{\tau}\right) \approx-\frac{A_{\tau}+\frac{2 \cos \vartheta_{\tau}}{1+\cos ^{2} \vartheta_{\tau}} A_{e}}{1+\frac{2 \cos \vartheta_{\tau}}{1+\cos ^{2} \vartheta_{\tau}} A_{e} A_{\tau}} .
$$

From this observable, one can extract information on the couplings $A_{\tau}$ and $A_{e}$, simultaneously.

In Figure 8 (left) we show the distribution of $P_{\tau}$ in the cosine of the scattering angle at the $Z$ peak in the Born and 1-loop (weak, QED, and EW) approximations. The same conventions as in previous sections are applied for the shifts $\Delta P_{\tau}$. The shift due to pure QED RCs is approximately a constant close to zero. But one can see that this observable is very sensitive to the presence of weak-interaction corrections.

In the presence of initial beams polarization the expression depends on $P_{\text {eff }}$ :

$$
P_{\tau}(\cos \vartheta) \approx-\frac{A_{\tau}\left(1-A_{e} P_{\mathrm{eff}}\right)+\frac{2 \cos \vartheta_{\tau}}{\left(1+\cos ^{2} \vartheta_{\tau}\right)}\left(A_{e}-P_{\mathrm{eff}}\right)}{\left(1-A_{e} P_{\mathrm{eff}}\right)+\frac{2 \cos \vartheta_{\tau}}{\left(1+\cos ^{2} \vartheta_{\tau}\right)} A_{\tau}\left(A_{e}-P_{\mathrm{eff}}\right)} .
$$

which can be reduced to the short form neglecting the $A_{e} A_{\tau}$ and $A_{e} P_{\text {eff }}$ terms:

$$
P_{\tau}\left(\cos \vartheta_{\tau}\right) \approx-A_{\tau}-\frac{2 \cos \vartheta_{\tau}}{\left(1+\cos ^{2} \vartheta_{\tau}\right)}\left(A_{e}-P_{\text {eff }}\right) .
$$

The influence of the initial particle polarization on $P_{\tau}$ at the $Z$ peak is demonstrated in the Figure 8 (right). For comparison the unpolarized and two polarized cases (13) as functions of $\cos \vartheta_{\tau}$ are shown. It is seen that the behavior of $P_{\tau}$ depends on the polarization set choices very much, note that it even changes the sign for the $P_{2}$ case. The corresponding shifts $\Delta P_{\tau}$ also strongly depend on the initial beam polarization degrees and change the shape accordingly (note the maximum for $P_{1}$ ).

In Figure 9 we show the dependence of $P_{\tau}$ on the c.m.s. energy in the Born and 1-loop approximations (weak, QED, and EW). We see that at energies above the $Z$ resonance, both weak and QED radiative corrections to $P_{\tau}$ are large and considerable cancellations happen between their contributions. Note that theoretical uncertainties in weak and QED RCs are not correlated, so it is necessary to take into account higher-order effects to reduce the resulting uncertainty in the complete 1-loop result for $P_{\tau}$ at high energies. 

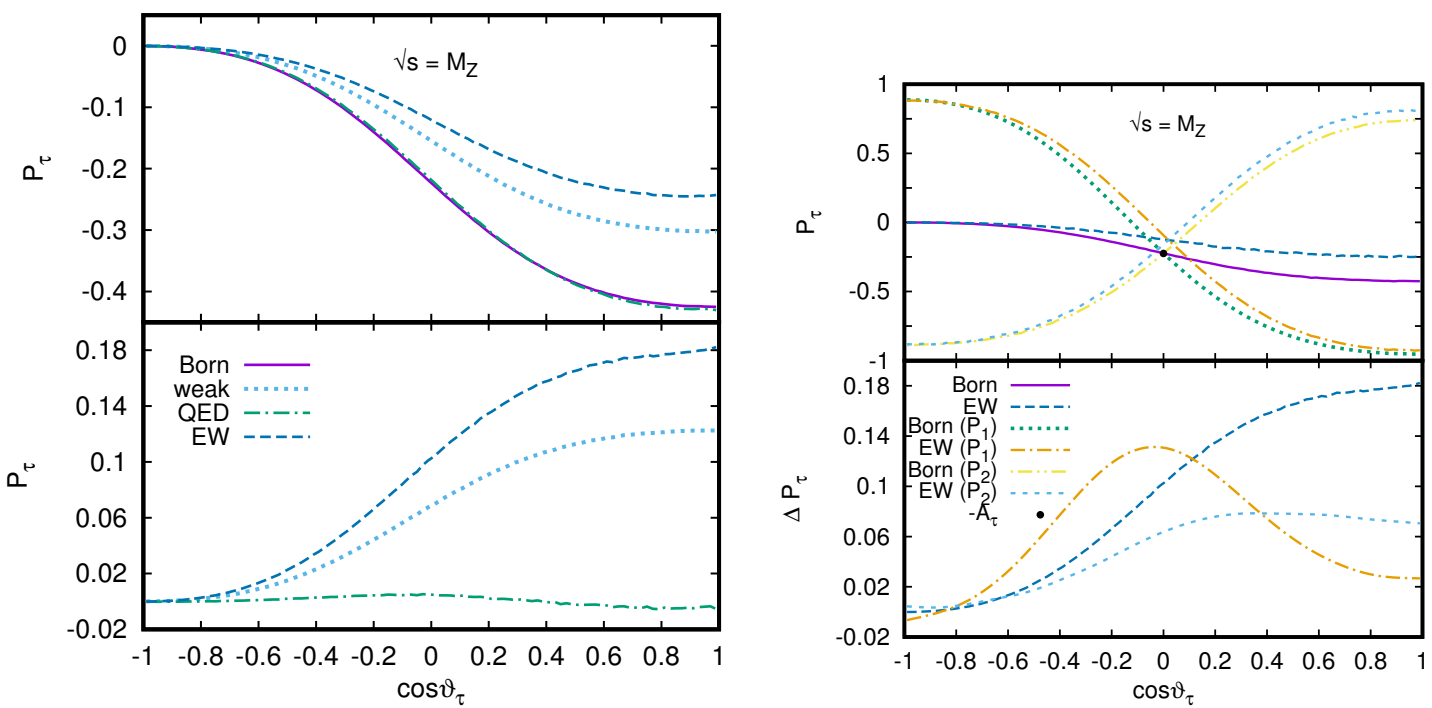

Figure 8. (Left) The $P_{\tau}$ polarization in the Born and 1-loop (weak, pure QED, and EW) approximations as a function of $\cos \vartheta_{\tau}$ at $\sqrt{s}=M_{z}$. (Right) The $P_{\tau}$ polarization for unpolarized and polarized cases with (13) degrees of initial beam polarizations in the Born and EW 1-loop approximations vs. cosine of the final $\tau$ lepton scattering angle at the $Z$ peak.
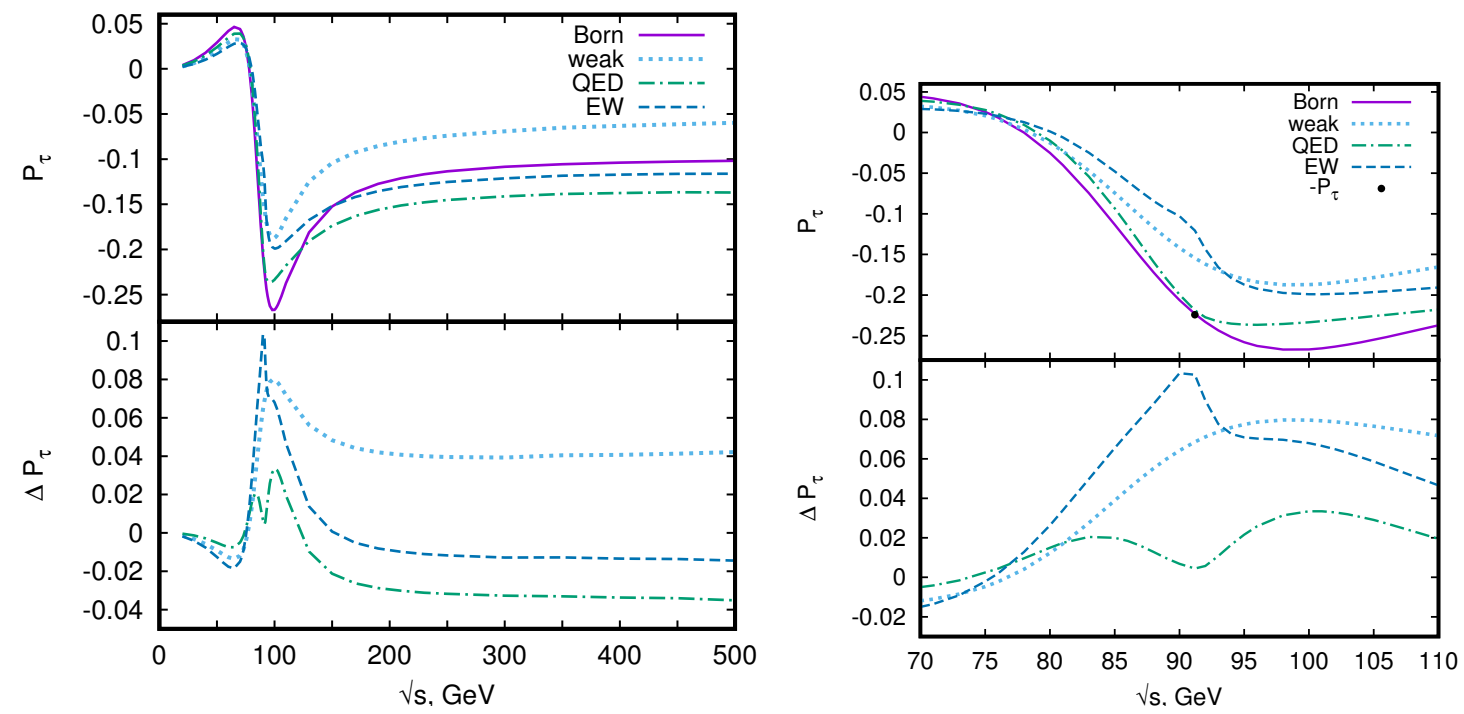

Figure 9. (Left) The $P_{\tau}$ polarization in the Born and 1-loop (weak, pure QED, and EW) approximations and $\Delta P_{\tau}$ vs. c.m.s. energy in a wide range; (Right) the same for the $Z$ peak region. The black dot indicates the value $P_{\tau}$ at the $Z$ resonance.

In Figure 10 we show $P_{\tau}$ in the Born and 1-loop EW approximations for different sets of beam polarization degrees in a narrow bin around the $Z$ resonance. The beam polarizations sets $P_{1}$ and $P_{2}$ are defined in Equation (13). One can see that the energy dependence of $P_{\tau}$ is strongly affected by a beam polarization choice outside the $Z$ peak region. The same concerns the size of radiative corrections to $P_{\tau}$, which are represented on the lower plot.

\section{Summary for $P_{\tau}$}

The $P_{\tau}$ asymmetry is very sensitive to weak-interaction corrections and to the polarization degrees of the initial beams. Near the $Z$ resonance the value of theoretical uncertainty of $P_{\tau}$ is determined by the interplay of uncertainties of rather large contributions pure QED and weak radiative corrections. 


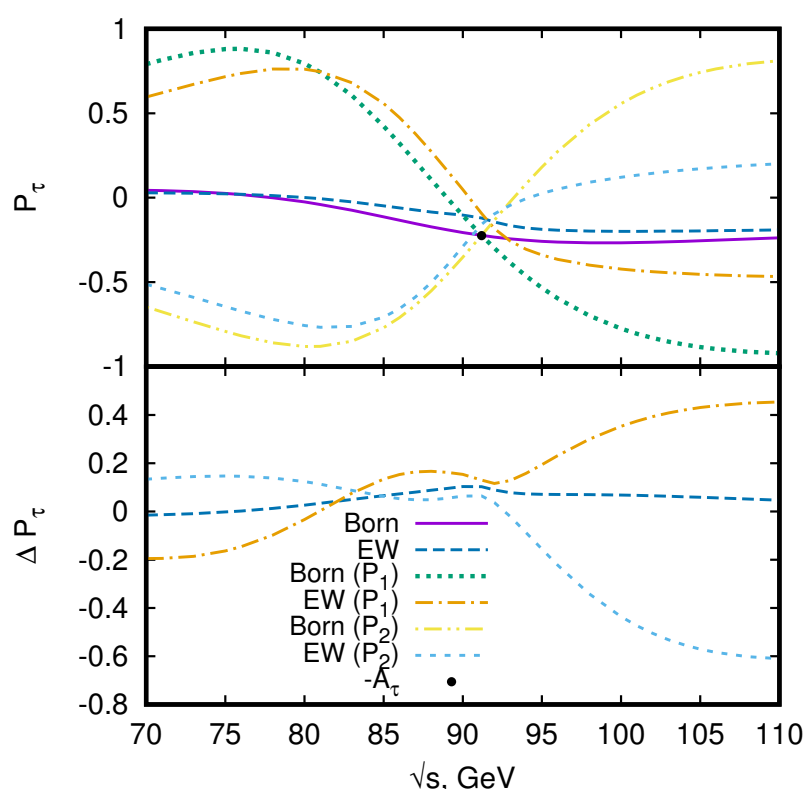

Figure 10. The $P_{\tau}$ polarization for (13) degrees of the initial beam polarizations in the Born and 1-loop EW approximations vs. c.m.s. energy in the $Z$ peak region.

\section{Conclusions}

New opportunities of the future $e^{+} e^{-}$colliders: GigaZ options and new energy scale up to several $\mathrm{TeV}$ require modern tools for high-precision theoretical calculations of observables. We investigated $A_{\mathrm{LR}}, A_{\mathrm{FB}}$ and $A_{\mathrm{LRFB}}$ for $e^{+} e^{-} \rightarrow \mu^{+} \mu^{-}$channel and polarization $P_{\tau}$ for the final state in $e^{+} e^{-} \rightarrow \tau^{+} \tau^{-}$ channel on the $Z$ resonance and in the high energy region up to $500 \mathrm{GeV}$ by using MCSANC. We evaluated the resulting shifts of asymmetries at the Born and EW levels of accuracy in different EW schemes. The numerical results presented above for pure QED, weak, and complete EW radiative corrections show an interplay between the weak and QED contributions to asymmetries. This fact indicates the necessity to consider those contributions always in combined way.

Asymmetries in $e^{+} e^{-}$annihilation processes provide a powerful tool for investigation of symmetries between three fermion generations. By studying all available asymmetries, one can extract parameters of weak interactions in the neutral current for all three charged leptons. So, by comparing the parameters it will be possible to verify the lepton universality hypothesis at a new level of precision.

Hypothetical extra neutral $Z^{\prime}$ vector bosons [29] can contribute to the processes of $e^{+} e^{-}$ annihilation. For example, effects of Kaluza-Klein excited vector bosons in the gauge Higgs unification on $e^{+} e^{-}$annihilation cross sections were considered in [30,31]. Since the new bosons can have couplings to left and right fermions being different from the SM ones, the asymmetries (especially with polarized beams) can help a lot in search for such $Z^{\prime}$ bosons.

At the FCC-ee we have experimental precision $\operatorname{tag}$ in the $\sin ^{2} \vartheta_{W}^{\text {eff }}$ measurement of the order of $5 \times 10^{-6}$, which means more than a thirty-fold improvement with respect to the current precision of $1.6 \times 10^{-4}$. This is due to a factor of several hundred improvement on statistical errors and because of a considerable improvement in particle identification and vertexing. In order to provide theoretical predictions for the considered asymmetries with sufficiently small uncertainties which would not spoil the precision of the future experiments besides the complete 1-loop EW radiative corrections presented here we need: 
- $\quad$ higher order pure QED corrections preferably with resummation;

- higher order (electro)weak corrections;

- taking into account perturbative and nonperturbative quantum chromodynamics (QCD) effects in RCs;

- Monte Carlo event generators and integrators which ensure the required technical precision.

Challenges in calculations of higher order QED effects for FCC-ee were discussed in Ref. [32]. The complete two-loop electroweak corrections in the vicinity of the $Z$ boson peak have been presented in [33]. More details on challenges for high-precision theoretical calculations for future $e^{+} e^{-}$colliders can be found in $[34,35]$.

Author Contributions: Conceptualization, A.A., S.B. and L.K.; methodology, A.A., S.B. and L.K.; software, A.A., S.B. and L.K.; validation, A.A., S.B. and L.K.; formal analysis, A.A., S.B. and L.K.; investigation, A.A., S.B. and L.K.; resources, A.A., S.B. and L.K.; data curation, A.A., S.B. and L.K.; writing-original draft preparation, A.A., S.B. and L.K.; writing-review and editing, A.A., S.B. and L.K. The authors claim to have contributed equally and significantly in this paper. All authors have read and agreed to the published version of the manuscript.

Funding: This research was funded by RFBR grant 20-02-00441.

Acknowledgments: The authors are grateful to Ya. Dydyshka, R. Sadykov, V. Yermolchyk, and A. Sapronov for fruitful discussions and numerical cross checks, and to A. Kalinovskaya for the help with preparation of the manuscript.

Conflicts of Interest: The authors declare no conflict of interest.

\section{Abbreviations}

The following abbreviations are used in this manuscript:

$\begin{array}{ll}\text { SM } & \text { Standard Model } \\ \text { QED } & \text { quantum electrodynamics } \\ \text { QCD } & \text { quantum chromodynamics } \\ \text { EW } & \text { electroweak } \\ \text { RCs } & \text { radiative corrections } \\ \text { FB } & \text { forward-backward } \\ \text { LR } & \text { left-right } \\ \text { c.m.s. } & \text { center-of-mass system }\end{array}$

\section{References}

1. Fujii, K.; Grojean, C.; Peskin, M.E.; Barklow, T.; Gao, Y.; Kanemura, S.; Kim, H.; List, J.; Nojiri, M.; Perelstein, M.; et al. Tests of the Standard Model at the International Linear Collider. arXiv 2019, arXiv:1908.11299.

2. A Multi-TeV Linear Collider Based on CLIC Technology: CLIC Conceptual Design Report; SLAC National Accelerator Lab.: Menlo Park, CA, USA, 2012. [CrossRef]

3. Moortgat-Pick, G. The Role of polarized positrons and electrons in revealing fundamental interactions at the linear collider. Phys. Rep. 2008, 460, 131-243. [CrossRef]

4. The International Linear Collider Technical Design Report-Volume 2: Physics. arXiv 2013, arXiv:1306.6352.

5. Bambade, P.; Barklow, T.; Behnke, T.; Berggren, M.; Brau, J.; Burrows, P.; Denisov, D.; Faus-Golfe, A.; Foster, B.; Fujii, K.; et al. The International Linear Collider: A Global Project. arXiv 2019, arXiv:1903.01629.

6. Abada, A. FCC-ee: The Lepton Collider: Future Circular Collider Conceptual Design Report Volume 2. Eur. Phys. J. ST 2019, 228, 261-623. [CrossRef]

7. Ahmad, M.; Alves, D.; An, H.; An, Q.; Arhrib, A.; Arkani-Hamed, N.; Ahmed, I.; Bai, Y.; Ferroli, R.B.; Ban, Y.; et al. CEPC-SPPC Preliminary Conceptual Design Report. 1. Physics and Detector. Preprint IHEP-CEPC-DR-2015-01. 2015. Available online: https:/ /inspirehep.net/literature/1395734 (accessed on 1 June 2020).

8. Bhupal Dev, P.; Djouadi, A.; Godbole, R.; Muhlleitner, M.; Rindani, S. Determining the CP properties of the Higgs boson. Phys. Rev. Lett. 2008, 100, 051801. [CrossRef]

9. Hagiwara, K.; Ma, K.; Yokoya, H. Probing CP violation in $e^{+} e^{-}$production of the Higgs boson and toponia. JHEP 2016, 6, 48. [CrossRef] 
10. Ma, K. Enhancing CP Measurement of the Yukawa Interactions of Top-Quark at $e^{-} e^{+}$Collider. Phys. Lett. B 2019, 797, 134928. [CrossRef]

11. Erler, J.; Heinemeyer, S.; Hollik, W.; Weiglein, G.; Zerwas, P. Physics impact of GigaZ. Phys. Lett. B 2000, 486, 1389-1402. [CrossRef]

12. Grunewald, M. Experimental tests of the electroweak standard model at high-energies. Phys. Rep. 1999, 322, 125-346. [CrossRef]

13. Schael, S. Precision electroweak measurements on the $Z$ resonance. Phys. Rep. 2006, 427, 257-454. [CrossRef]

14. Mnich, J. Experimental tests of the standard model in $e^{+} e^{-} \rightarrow \mathrm{f}$ anti-f at the $Z$ resonance. Phys. Rep. 1996, 271, 181-266. [CrossRef]

15. Arbuzov, A.; Awramik, M.; Czakon, M.; Freitas, A.; Grunewald, M.; Monig, K.; Riemann, S.; Riemann, T. ZFITTER: A Semi-analytical program for fermion pair production in $e^{+} e^{-}$annihilation, from version 6.21 to version 6.42. Comput. Phys. Commun. 2006, 174, 728-758. [CrossRef]

16. Montagna, G.; Nicrosini, O.; Piccinini, F.; Passarino, G. TOPAZ0 4.0: A New version of a computer program for evaluation of deconvoluted and realistic observables at LEP-1 and LEP-2. Comput. Phys. Commun. 1999, 117, 278-289. [CrossRef]

17. Bondarenko, S.; Dydyshka, Y.; Kalinovskaya, L.; Sadykov, R.; Yermolchyk, V. One-loop electroweak radiative corrections to lepton pair production in polarized electron-positron collisions. arXiv 2020, arXiv:2005.04748.

18. Andonov, A.; Arbuzov, A.; Bardin, D.; Bondarenko, S.; Christova, P.; Kalinovskaya, L.; Nanava, G.; von Schlippe, W. SANCscope-v.1.00. Comput. Phys. Commun. 2006, 174, 481-517. [CrossRef]

19. Arbuzov, A.; Bondarenko, S.; Dydyshka, Y.; Kalinovskaya, L.; Rumyantsev, L.; Sadykov, R.; Yermolchyk, V. Electron-positron annihilation processes in MCSANCee. CERN Yellow Rep. Monogr. 2020, 3, $213-216$. [CrossRef]

20. Arbuzov, A.; Bardin, D.; Bondarenko, S.; Christova, P.; Kalinovskaya, L.; Klein, U.; Kolesnikov, V.; Rumyantsev, L.; Sadykov, R.; Sapronov, A. Update of the MCSANC Monte Carlo integrator, v. 1.20. JETP Lett. 2016, 103, 131-136. [CrossRef]

21. Blondel, A. A Scheme to Measure the Polarization Asymmetry at the Z Pole in LEP. Phys. Lett. B 1988, 202, 145. [CrossRef]

22. Janot, P. Direct measurement of $\alpha_{Q E D}\left(m_{Z}^{2}\right)$ at the FCC-ee. JHEP 2016, 2, 53. [CrossRef]

23. Jadach, S.; Yost, S. QED Interference in Charge Asymmetry Near the Z Resonance at Future Electron-Positron Colliders. Phys. Rev. D 2019, 100, 013002. [CrossRef]

24. Arbuzov, A.; Bardin, D.; Leike, A. Analytic final state corrections with cut for $\mathrm{e}+\mathrm{e}-\longrightarrow$ massive fermions. Mod. Phys. Lett. A 1992, 7, 2029-2038. [CrossRef]

25. Blondel, A.; Lynn, B.; Renard, F.; Verzegnassi, C. Precision Measurements of Final State Weak Coupling from Polarized Electron-Positron Annihilation. Nucl. Phys. B 1988, 304, 438-450. [CrossRef]

26. Jadach, S.; Was, Z.; Decker, R.; Kuhn, J.H. The tau decay library TAUOLA: Version 2.4. Comput. Phys. Commun. 1993, 76, 361-380. [CrossRef]

27. Jadach, S.; Ward, B.; Was, Z. The Monte Carlo program KORALZ, version 4.0, for the lepton or quark pair production at LEP/SLC energies. Comput. Phys. Commun. 1994, 79, 503-522. [CrossRef]

28. Jadach, S.; Ward, B.; Was, Z. The Monte Carlo program KORALZ, for the lepton or quark pair production at LEP/SLC energies: From version 4.0 to version 4.04. Comput. Phys. Commun. 2000, 124, 233-237. [CrossRef]

29. Langacker, P. The Physics of Heavy Z' Gauge Bosons. Rev. Mod. Phys. 2009, 81, 1199-1228. [CrossRef]

30. Funatsu, S.; Hatanaka, H.; Hosotani, Y.; Orikasa, Y. Distinct signals of the gauge-Higgs unification in $e^{+} e^{-}$ collider experiments. Phys. Lett. B 2017, 775, 297-302. [CrossRef]

31. Funatsu, S. Forward-backward asymmetry in the gauge-Higgs unification at the International Linear Collider. Eur. Phys. J. C 2019, 79, 854. [CrossRef]

32. Jadach, S.; Skrzypek, M. QED challenges at FCC-ee precision measurements. Eur. Phys. J. C 2019, $79,756$. [CrossRef]

33. Dubovyk, I.; Freitas, A.; Gluza, J.; Riemann, T.; Usovitsch, J. Electroweak pseudo-observables and Z-boson form factors at two-loop accuracy. JHEP 2019, 8, 113. [CrossRef] 
34. Blondel, A.; Freitas, A.; Gluza, J.; Riemann, T.; Heinemeyer, S.; Jadach, S.; Janot, P. Theory Requirements and Possibilities for the FCC-ee and other Future High Energy and Precision Frontier Lepton Colliders. arXiv 2019, arXiv:1901.02648.

35. Blondel, A.; Gluza, J.; Jadach, S.; Janot, P.; Riemann, T. (Eds.) Theory for the FCC-ee: Report on the 11th FCC-ee Workshop Theory and Experiments; Vol. 3/2020; CERN Yellow Reports: Monographs; CERN: Geneva, Switzerland, 2019. [CrossRef] article distributed under the terms and conditions of the Creative Commons Attribution (CC BY) license (http:/ / creativecommons.org/licenses/by/4.0/). 